\title{
Prohibitin-2 Depletion Unravels Extra-Mitochondrial Functions at the Kidney Filtration Barrier
}

\author{
Christina Ising, ${ }^{* \dagger}$ Puneet Bharill, ${ }^{*}$ Sibylle Brinkkoetter, ${ }^{*}$ Sebastian Brähler, ${ }^{* *}$ Christina Schroeter, ${ }^{*}$ Sybille Koehler, * \\ Henning Hagmann, ${ }^{*}$ Carsten Merkwirth, ${ }^{\S \Psi}$ Martin Höhne, ${ }^{*}$ Roman U. Müller, ${ }^{*}$ Francesca Fabretti, ${ }^{*}$ Bernhard Schermer, ${ }^{*} \|$ \\ Wilhelm Bloch, ${ }^{* *}$ Dontscho Kerjaschki, ${ }^{\dagger \dagger}$ Christine E. Kurschat, ${ }^{* \|}$ Thomas Benzing, ${ }^{* \|}$ and Paul T. Brinkkoetter*
}

\begin{abstract}
From the Department II of Internal Medicine and Center for Molecular Medicine Cologne* and the Institute for Genetics, ${ }^{\S}$ University of Cologne, Cologne, Germany; the Department of Neurology, ${ }^{\dagger}$ Hope Center for Neurological Disorders, Charles F. and Joanne Knight Alzheimer's Disease Research Center, and the Department of Pathology and Immunology, ${ }^{\ddagger}$ Washington University School of Medicine, St. Louis, Missouri; the Howard Hughes Medical Institute, ${ }^{\natural}$ University of California Berkeley, Berkeley, California; the Cologne Cluster of Excellence on Cellular Stress Responses in Ageing-Associated Diseases $(C E C A D)$ and Systems Biology of Ageing Cologne (Sybacol) $)^{\|}$and the Institute of Cardiovascular Research and Sport Medicine, ** Department of Molecular and Cellular Sport Medicine, German Sport University Cologne, Cologne, Germany; and the Clinical Institute of Pathology, ${ }^{\dagger \dagger}$ Medical University of Vienna, Vienna, Austria
\end{abstract}

Accepted for publication

December 22, 2015.

Address correspondence to Paul T. Brinkkoetter, M.D., Department II of Internal Medicine, University of Cologne, Kerpener Str. 62, 50937 Cologne, Germany. E-mail: paul. brinkkoetter@uk-koeln.de.

\begin{abstract}
Mitochondrial fusion is essential for maintenance of mitochondrial function and requires the prohibitin ring complex subunit prohibitin-2 (PHB2) at the mitochondrial inner membrane. Loss of the stomatin/ PHB/flotillin/HflK/C (SPFH) domain containing protein PHB2 causes mitochondrial dysfunction and defective mitochondria-mediated signaling, which is implicated in a variety of human diseases, including progressive renal disease. Here, we provide evidence of additional, extra-mitochondrial functions of this membrane-anchored protein. Immunofluorescence and immunogold labeling detected PHB2 at mitochondrial membranes and at the slit diaphragm, a specialized cell junction at the filtration slit of glomerular podocytes. PHB2 coprecipitated with podocin, another SPFH domain-containing protein, essential for the assembly of the slit diaphragm protein-lipid supercomplex. Consistent with an evolutionarily conserved extra-mitochondrial function, the ortholog of PHB2 in Caenorhabditis elegans was also not restricted to mitochondria but colocalized with the mechanosensory complex that requires the podocin ortholog MEC2 for assembly. Knockdown of phb-2 partially phenocopied loss of mec-2 in touch neurons of the nematode, resulting in impaired gentle touch sensitivity. Collectively, these data indicate that, besides its established role in mitochondria, PHB2 may have an additional function in conserved protein-lipid complexes at the plasma membrane. (Am J Pathol 2016, 186: 1128-1139; http://dx.doi.org/10.1016/j.ajpath.2015.12.018)
\end{abstract}

Among other diseases, the renal involvement of mitochondrial disorders often manifests clinically with podocyte dysfunction, resulting in early onset of steroid-resistant nephrotic syndrome. However, the mitochondrial contribution to disease, the high-energy demand of podocytes, and the underlying mitochondrial signaling pathways are only incompletely understood.

The structural proteins prohibitin-1 (PHB1) and prohibitin-2 (PHB2) reside within the mitochondrial inner membrane where they directly interact and form multimeric ring complexes to stabilize each other. ${ }^{1-3}$ Proposed models support a function of PHBs as membrane scaffolds that are required for the maintenance of cristae structures. ${ }^{4,5} \mathrm{PHBs}$ may also affect the lipid distribution in the mitochondrial inner membrane, ${ }^{6}$ serve a scaffolding function during biogenesis and assembly of oxidative phosphorylation system complexes, ${ }^{7}$ and influence the susceptibility toward apoptotic cell death. ${ }^{5,8,9}$ Additional functions of PHBs are attributed to other cellular compartments, for example, the

\footnotetext{
Supported by a fellowship from the International Graduate School in Development Health and Disease at the University of Cologne, Germany (C.I.); the Deutsche Forschungsgemeinschaft grants BR2955/4-1 (P.T.B.), SCHE1562/2-1 (B.S.), SFB635 (T.B.), and SFB829 (T.B.); and Köln Fortune Program of the University of Cologne, Germany (P.T.B.)

Disclosures: None declared.
} 
plasma membrane ${ }^{10,11}$ and nucleus, ${ }^{12,13}$ and comprise roles in cell cycle progression, regulation of transcription, and cell surface signaling. However, many of the underlying intracellular pathways involved in the action of PHBs beyond mitochondria are a matter of a highly controversial debate. $^{12-16}$

Podocyte-specific loss of PHB2 and subsequently PHB1 led to an early onset of proteinuria in 3-week-old mice, renal failure, and, eventually, premature death after 4 to 5 weeks. ${ }^{17}$ Moreover, depletion of PHB2 resulted in fragmentation of the mitochondrial network but did not affect oxidative phosphorylation function, oxygen consumption, or production of reactive oxygen species. Although inhibiting either the insulin or mammalian target of rapamycin signaling pathways delayed the onset of glomerular disease in Phb2-deficient animals, it did not affect the onset and magnitude of proteinuria. ${ }^{17}$

Proteinuria is closely linked to loss of proteins that contribute to the formation of the podocyte slit diaphragm such as podocin, the protein mutated in autosomal-recessive steroid-resistant nephrotic syndrome. ${ }^{18}$ Podocin and PHB1 and PHB2 are stomatin/PHB/flotillin/HflK/C (SPFH) domain-containing proteins. Therefore, we hypothesized additional, nonmitochondrial functions of PHB $1 / 2$ at this specialized cell-cell contact.

Here, we show that PHBs are required to maintain healthy podocyte foot processes. PHB2 and possibly PHB1 localize to podocyte foot processes where they form a protein complex with podocin. By using the model organism Caenorhabditis elegans we show that phb-1/phb-2 deficiency disturbs the function of the mechanosensory complex, which is highly similar to the multiprotein complex at the slit diaphragm. These results argue for extramitochondrial functions of PHB1 and PHB2 in podocytes.

\section{Materials and Methods}

\section{Mouse Models}

Mice in which exons 3 and 4 of the $P h b 2$ gene are flanked by two loxP sequences ${ }^{5}$ were mated to NPHS2.Cre mice ${ }^{19}$ to generate podocyte-specific $P h b 2$ knockout mice $P h b 2^{f / f}$; NPHS2.Cre ${ }^{\mathrm{tg} / \mathrm{wt}}\left(P h b 2^{p k o}\right), P h b 2^{f l w t} ; \mathrm{NPHS} 2 . \mathrm{Cre}^{\mathrm{tg} / \mathrm{wt}}\left(P h b 2^{h e t}\right)$, or $P h b 2^{f l / f}$;NPHS2.Cre ${ }^{\mathrm{wt} / \mathrm{wt}}\left(P h b 2^{f / f l}\right)$ mice. All animals were backcrossed onto the C57/B16 background for at least 10 generations. Mice were housed according to the standardized specific pathogen-free conditions in the University of Cologne animal facility. The State Agency for Nature, Environment and Consumer Protection North Rhine-Westphalia reviewed and approved the experimental protocol.

\section{Electron Microscopy}

Mice were perfused with electron microscopy fixation buffer (4\% paraformaldehyde and $2 \%$ glutaraldehyde in 0.1 $\mathrm{mol} / \mathrm{L}$ sodium cacodylate, $\mathrm{pH}$ 7.4) and the kidneys were post-fixed in the same buffer for 2 weeks at $4^{\circ} \mathrm{C}$. Samples were osmicated with $1 \% \mathrm{OsO}_{4}$ in $0.1 \mathrm{~mol} / \mathrm{L}$ cacodylate and dehydrated in a graduated ethanol series. Infiltration with Epon and flat embedding were performed according to standard procedures. Thin $(30 \mathrm{~nm})$ cross-sections were taken on an Ultracut UCT ultramicrotome (Reichert, Heidelberg, Germany). The sections were stained with $1 \%$ aqueous uranylic acetate and lead citrate and examined with a Zeiss EM 902 electron microscope (Carl Zeiss MicroImaging GmbH, Oberkochen, Germany). For immunogold labeling, fixed samples of mouse or human kidneys were embedded in Lowicryl K4M resin (Electron Microscopy Sciences, Hatfield, PA), and ultrathin sections were labeled by an indirect immunogold protocol (Table 1) as described previously. ${ }^{20}$ Images were further processed with Adobe Photoshop CS4 version 11.0.0.0. (Adobe Systems, San Jose, CA).

\section{Cell Culture}

Conditionally immortalized podocytes were generated as previously described ${ }^{21,22}$ and cultured in RPMI media supplemented with $10 \%$ fetal bovine serum. Differentiation was induced by culturing the cells at $37^{\circ} \mathrm{C}$ for 14 days on Primaria plastic plates (BD Biosciences, San Jose, CA) in the absence of interferon- $\gamma$. All experiments were performed with differentiated cells. Human embryonic kidney cell line 293T (HEK293T) were maintained in Dulbecco's modified Eagle's medium supplemented with $10 \%$ fetal bovine serum.

\section{Antibody Generation}

Bacterially expressed and affinity-purified full-length Histagged PHB1 and PHB2 were used to immunize mice,

Table 1 Antibodies Used in This Study

\begin{tabular}{|c|c|c|c|}
\hline Name & Company & $\begin{array}{l}\text { Host } \\
\text { species }\end{array}$ & Dilution \\
\hline Anti-Flag & Sigma-Aldrich & Rabbit & $\begin{array}{l}1 \mu \mathrm{g} \text { for IP, } \\
1: 2000 \text { for WB }\end{array}$ \\
\hline Anti-Flag(M2) & Sigma-Aldrich & Mouse & Coupled to beads \\
\hline Anti-pan14-3-3 & $\begin{array}{l}\text { Santa Cruz } \\
\text { Biotechnology } \\
\text { (Santa Cruz, CA) }\end{array}$ & Rabbit & 1:1000 for WB \\
\hline Anti-PHB1 & $\begin{array}{l}\text { BioLegend } \\
\quad \text { (San Diego, CA) }\end{array}$ & Rabbit & 1:1000 for $W B$ \\
\hline Anti-PHB2 & $\begin{array}{l}\text { Hybridoma } \\
\text { supernatant } \\
\text { fluid }\end{array}$ & Mouse & Undiluted for IF \\
\hline Anti-PHB2 & BioLegend & Rabbit & $\begin{array}{l}\text { 1:10 for } \\
\text { immunogold, } \\
\text { 1:1000 for WB }\end{array}$ \\
\hline Anti-podocin & Sigma-Aldrich & Rabbit & $1: 100$ for IF \\
\hline Anti-V5 & Serotec & Mouse & $1 \mu \mathrm{g}$ for IP \\
\hline Anti-V5 & $\begin{array}{l}\text { Millipore } \\
\quad \text { (Billerica, MA) }\end{array}$ & Rabbit & $1: 2000$ for $W B$ \\
\hline
\end{tabular}

IF, immunofluorescence; IP, immunoprecipitation; PHB, prohibitin; WB, Western blot analysis. 
following a standard immunization protocol. ${ }^{23}$ Fusions resulted in the generation of $>40$ PHB1- and PHB2detecting monoclonal antibodies. Positive clones were identified by enzyme-linked immunosorbent assay. Briefly, 200 ng His-tagged PHB1, PHB2, or a control protein were coupled to the wells of a Nunc immunosorb 8-strip. After blocking with $1 \%$ bovine serum albumin (Sigma-Aldrich, St. Louis, MO) in phosphate-buffered saline, the hybridoma supernatant fluid was applied to the wells. After incubation with a horseradish peroxidase-conjugated anti-mouse antibody (Dianova, Hamburg, Germany) a tetramethylbenzidene substrate diluted in sodium acetate and hydrogen peroxide was applied to every well and incubated for 15 minutes before termination of the reaction with hydrochloric acid. Absorption at $450 \mathrm{~nm}$ was measured with a Mithras LB 940 Microplate Reader (Berthold, Bad Wildbad, Germany). Positive clones were subcloned and further validated by immunoblotting and immunofluorescence staining before antibodies were concentrated with Protein G columns.

\section{Immunofluorescence on Cells and Human/Mouse Tissue}

Human tissue samples were obtained from tumor nephrectomy samples after obtaining written consent as approved by the local ethic committee. For immunofluorescence staining of immortalized mouse podocytes, ${ }^{21,22}$ the cells were seeded on coverslips and differentiated at $37^{\circ} \mathrm{C}$ for 14 days before incubation with 250 or $500 \mathrm{nmol} / \mathrm{L}$ MitoTracker for 15 minutes at $37^{\circ} \mathrm{C}$ and subsequent fixation and staining. Cells or frozen tissues were fixed in $4 \%$ paraformaldehyde before incubation in blocking solution (5\% normal donkey serum in PBS with $0.1 \%$ Triton X-100) and subsequently incubated with primary (Table 1) and secondary antibodies. Tissues were mounted in Prolong Gold antifade with DAPI (Invitrogen, Carlsbad, CA). For experiments with blocking peptide, the primary PHB1/2 antibody was preincubated with or without recombinant PHB1 for 1 hour at room temperature. After centrifugation for 15 minutes, the supernatant fluid was used for staining. Images of cells were acquired with an Axiovert $200 \mathrm{~mol} / \mathrm{L}$ microscope/EC PlanNeofluar $63 \times / 1.30$ water immersion objective (all from Carl Zeiss MicroImaging $\mathrm{GmbH}$ ) and images of tissues with an LSM 710/Axioobserver Z1 confocal microscope $\times 40 / 1.1$ water immersion objective operated by ZEN 2009 software (all from Carl Zeiss MicroImaging GmbH, Oberkochen, Germany). Images were further processed with the use of ImageJ/Fiji software version 1.46 (NIH, Bethesda, MD; http://imagej.nih.gov/ij) and Adobe Photoshop CS4 version 11.0.0.0 (Adobe Systems).

\section{Co-Immunoprecipitation}

HEK293T cells were transiently transfected with a standard calcium phosphate method. ${ }^{24}$ One day after transfection cells were lyzed directly in a modified RIPA buffer (50 $\mathrm{mmol} / \mathrm{L}$ Tris-HCl, $\mathrm{pH} 7.4,1 \%$ Igepal CA-630, 0.25\% Nadeoxycholate, $150 \mathrm{mmol} / \mathrm{L} \mathrm{NaCl}, 1 \mathrm{mmol} / \mathrm{L}$ EDTA, 1 $\mathrm{mmol} / \mathrm{L}$ phenylmethylsulfonyl fluoride, $2 \mathrm{mmol} / \mathrm{L} \mathrm{Na}_{3} \mathrm{VO}_{4}$ ) for 15 minutes on ice. After centrifugation at 20,000 $\times g(15$ minutes, $4^{\circ} \mathrm{C}$ ) a small aliquot of each supernatant fluid was preserved and diluted with $2 \times$ SDS-PAGE sample buffer for Western blot analysis (ie, lysate). The remaining supernatant fluids were incubated with anti-FLAG(M2) antibody covalently coupled to agarose beads (Sigma-Aldrich) or with $1 \mu \mathrm{g}$ of V5 antibody (Serotec, Raleigh, NC) and 30 $\mu \mathrm{L}$ of protein-G-sepharose beads (GE Healthcare, Little Chalfont, UK). After extensive washing with lysis buffer, bound proteins were resolved by SDS-PAGE, blotted onto polyvinylidene difluoride membranes, and visualized with enhanced chemiluminescence after the incubation of the blots with the respective antibodies (Table 1).

For endogenous co-immunoprecipitation, glomeruli were isolated from wild-type mice as described previously. ${ }^{25}$ Isolated glomeruli were homogenized in $2 \mathrm{~mL}$ of modified RIPA buffer with 20 strokes in a dounce glass-glass homogenizer before sonication on ice ( 1.5 minutes, 0.5 -second pulses). The suspension was incubated on ice for $15 \mathrm{mi}-$ nutes. After centrifugation at $20,000 \times g\left(15\right.$ minutes, $\left.4^{\circ} \mathrm{C}\right)$ a small aliquot of the supernatant fluid was preserved and diluted with $5 \times$ SDS-PAGE sample buffer for Western blot analysis (ie, lysate). The remaining supernatant fluid was split into two tubes and incubated with either $1 \mu \mathrm{g}$ of podocin antibody (Sigma-Aldrich; host species: rabbit) and $30 \mu \mathrm{L}$ of protein-A-sepharose beads (GE Healthcare) or with $1 \mu \mathrm{g}$ of Flag antibody (Sigma-Aldrich; host species: rabbit) and $30 \mu \mathrm{L}$ of protein-A-sepharose beads (GE Healthcare). After extensive washing with lysis buffer, bound proteins were resolved by SDS-PAGE, blotted onto polyvinylidene difluoride membranes, and visualized with enhanced chemiluminescence after the incubation of the blots with the respective antibodies (Table 1).

\section{Velocity Gradient}

HEK293T cells were transiently transfected with two different Stealth siRNAs (Invitrogen) with the use of Lipofectamine 2000. Forty-eight hours after transfection with siRNA the cells were transfected with FLAG-tagged podocin. Twenty-four hours later each $10-\mathrm{cm}$ dish was lyzed in $500 \mathrm{~mL}$ of MBS buffer [ $25 \mathrm{mmol} / \mathrm{L} 2-(N$-morpholino)ethanesulfonic acid, $\mathrm{pH} 6.5 ; 150 \mathrm{mmol} / \mathrm{L} \mathrm{NaCl}]$ with $1 \%$ Triton X-100 by mixing on a vortex and incubation on ice for 20 minutes. After spinning for 10 minutes with $3000 \times g$, the supernatant fluid was mixed with SDS (final concentration $0.1 \%$ ) and incubated on ice for 20 minutes. After a 15-minute ultracentrifugation step $300 \mu \mathrm{L}$ of the supernatant fluid was loaded on top of a sucrose gradient (2 $\mathrm{mL} 60 \%$ sucrose solution layered with $250 \mu \mathrm{L} 40 \%$ to $5 \%$ sucrose solution, each in 5\% steps; sucrose was diluted in MBS buffer with $0.1 \%$ Triton X-100). The sucrose gradient was subjected to ultracentrifugation at $120,000 \times g$ for 16 


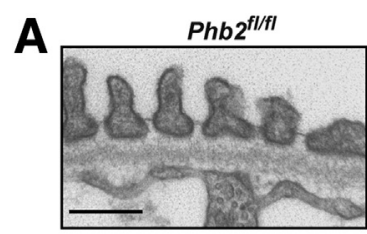

B

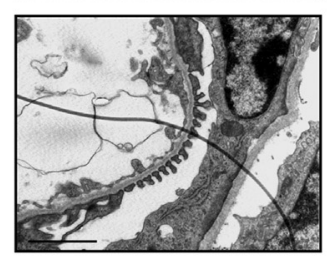

C

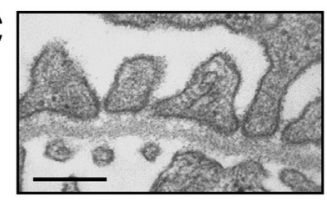

D

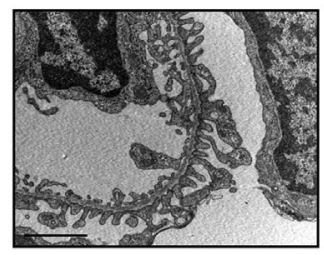

E

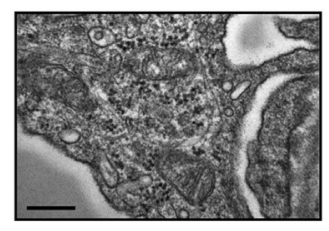

$\mathbf{F}$

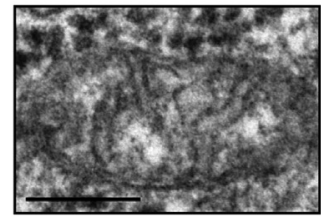

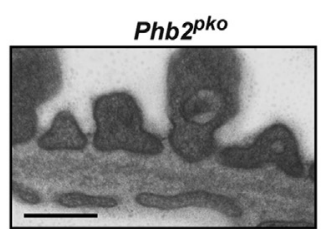
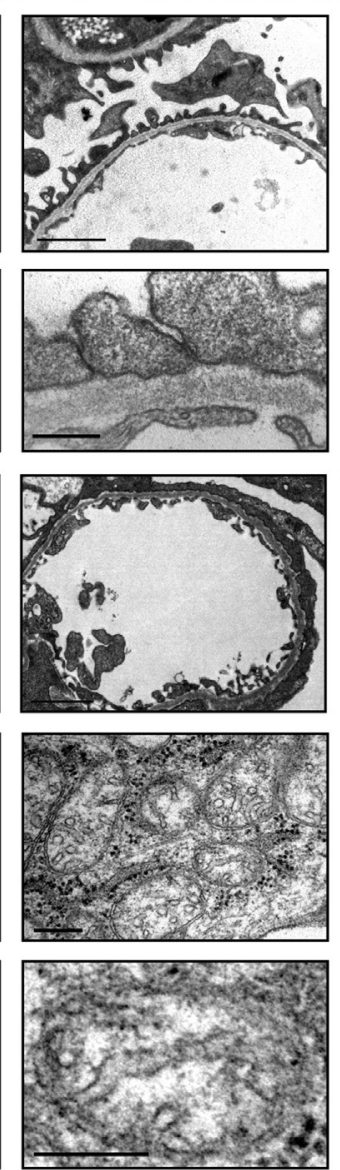
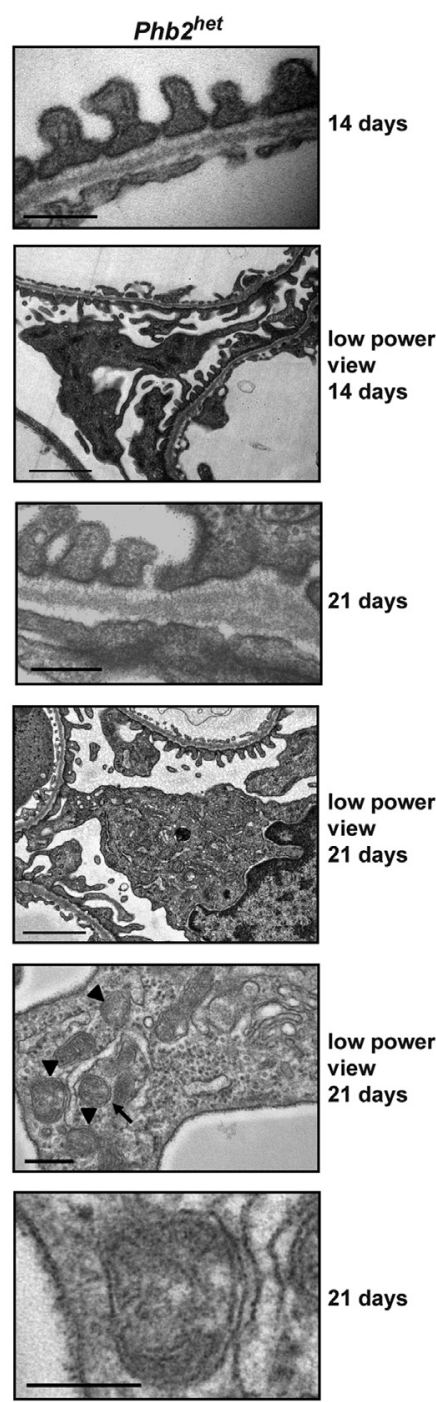

Figure 1 PHB2 is required to maintain structural integrity of podocyte foot processes and mitochondria. A: Slit diaphragm appearance analyzed in electron micrographs in 14-day-old $P h b 2^{f l f l}$ and $P h b 2^{p k o}$ mice. B: Low power view of foot process structure from 14-day-old mice of all three genotypes. C: Loss of slit diaphragm structure in 21-day-old $P h b 2^{\text {pko }}$ but not $P h b 2^{f l f f l}$ or Phb2 ${ }^{\text {het }}$ mice. D: Low-power view of foot process structure from 21-day-old mice of all three genotypes. E: Analysis of mitochondrial structure in low-power view of all three genotypes 21 days after birth shows disturbed mitochondrial cristae structures in $\mathrm{Phb2}^{\text {pko }}$ and partially also in $\mathrm{Phb2} 2^{\text {het }}$ mice (arrow indicates a normal mitochondrial structure; arrowheads indicate defective mitochondrial structures). F: High-power view of mitochondria from panel E. $n=3$ mice analyzed for all time points and genotypes. Scale bars: 0.3 $\mu \mathrm{m}(\mathbf{A}$ and $\mathbf{C}) ; 1 \mu \mathrm{m}$ (B, D, and $\mathbf{E}) ; 0.5 \mu \mathrm{m}(\mathbf{F})$. PHB, prohibitin. hours before $250 \mu \mathrm{L}$ fractions were collected and analyzed by SDS-PAGE and Western blot analysis (Table 1).

\section{Worm Strains}

We used TU3568 [sid-1(pk3321) him-5(e1490) V; lin15B(n744) X; uIs71 [pCFJ90(myo-2p::mCherry) + mec18p::sid-1]], a kind gift of Prof. Martin Chalfie (Columbia University, New York, NY). ${ }^{26}$ Because of an integrated array that contains mec-18p::sid-1 in a sid-1 mutant background, it provides efficient RNA interference (RNAi) only in touch receptor neurons. For microinjection of the

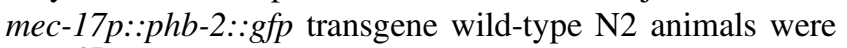
used. ${ }^{27}$ All strains were cultured at $20^{\circ} \mathrm{C}$.

\section{RNAi}

We performed RNAi by feeding as described previously ${ }^{27,28}$ with the use of the RNAi-sensitized background of TU3568 worms. RNAi clones corresponding to the target genes phb-1, phb-2, mec-2, and mev-1 were used for knockdown experiments. Empty RNAi vector L4440 was used as control RNAi, mec-2 as positive control, and mev-1, encoding another mitochondrial protein as control for unspecific RNAi effects on the basis of an impairment of the mitochondrial respiratory chain. All clones except phbl were picked from an RNAi feeding library constructed by the Ahringer laboratory at the Wellcome CRC Institute, University of Cambridge (Cambridge, United Kingdom) ${ }^{29}{ }^{29}$ To generate a $p h b 1$ RNAi clone, we PCR amplified a $0.8-\mathrm{kb}$ fragment with the use of N2 genomic DNA as template and primers published previously ${ }^{30}$ [Phb1-forward (5'CAATGTTGATGGAGGTCAACG-3') and Phb1-reverse $\left(5^{\prime}\right.$-GGTGACATTCTTGTTCTTGGC- $\left.3^{\prime}\right)$ ]. We cloned a $0.656-\mathrm{kb} \mathrm{NcoI} / \mathrm{SacI}$ fragment into the likewise digested L4440 vector. The construct was electroporated into HT115 bacteria. Bacteria that contained the different knockdown plasmids were seeded onto nematode growth mediumisopropyl-thio- $\beta$-d-galactoside plates. Plates were kept 16 hours at room temperature for generation of double-stranded RNA. Worm eggs were harvested by bleaching gravid adult animals in $0.5 \mathrm{~N} \mathrm{NaOH}$ and $2 \%$ to $4 \% \mathrm{NaOCl}$. The eggs were 
washed in M9 buffer, placed on the seeded plates, and grown at $20^{\circ} \mathrm{C}$. Young adult hermaphrodites were tested for touch sensation as described in Touch Assay below.

\section{Microinjection}

For germline injection, the $p h b-2: \because g f p$ plasmid was prepared via multisite gateway cloning (Invitrogen). Briefly, the promoter of mec-17 (mec-17p) was cloned from a C. elegans library (forward primer: 5'-GGGGACAACTTTGTATAGAAAAGTTGATTTTCTGAAATTACTATTA-3'; reverse primer: 5'-GGGGACTGCTTTTTTGTACAAACTTGTCATGATCGAATCGTCTCACAACT-3') and recombined into pDONR P4-P1R (Invitrogen) according to the manufacturer's protocol. $p h b-2$ was cloned without stop codon from the same library (forward primer: 5'-GGGGACAAGTTTGTACAAAAAAGCAGGCTTGGCGAAACAAGGGCAAGAAGC-3'; reverse primer: 5'-GGGGACCACTTTGTACAAGAAAGCTGGGTAGCGTCTTTTGTCGGTCAC-3') and recombined into pDONR 221 (Invitrogen) according to the manufacturer's protocol. Both, mec-17p and $p h b-2$ were recombined from the pENTR plasmids into pDEST MB14 to generate a plasmid that expressed PHB-2:GFP under control of the mec-17 promoter.

We generated lines that contained extrachromosomal arrays by injecting the $p h b-2: \because g f p$ plasmid at a concentration of $50 \mathrm{ng} / \mathrm{uL}$ mixed with pmyo-2::mCherry as a co-injection marker at the concentration of $5 \mathrm{ng} / \mathrm{uL}$ into wild-type (N2) worms. Single-positive transgenic F1 worms klnEx5 [pmec17::phb-2::gfp::unc-54 3'utr; pmyo-2::mCherry::unc-54 $\left.3^{\prime} u t r\right]$ were moved onto nematode growth medium plates and allowed to proliferate to form different lines.

\section{Touch Assay}

Animals were tested for response to gentle touch as described previously. ${ }^{31}$ Briefly, each animal was touched 10 times by a thin hair at the anterior part of the body. Stopping or backward movements were scored and shown as touch response of 10 touches. The data were generated in three independent experiments with the use of $\geq 15$ worms each time for each data point. Statistical evaluation was performed as described in Statistical Analysis.

\section{Immunofluorescence on Worms}

We performed antibody staining on mec-17p::phb-2::gfp transgenic worms with the use of a modified FinneyRuvkun protocol. ${ }^{32}$ Worms were prepared for antibody staining as described previously. ${ }^{33}$ Specimens were incubated with a rabbit antibody that detected the $\mathrm{N}$-terminus of MEC-2 (kind gift of Prof. Martin Chalfie; dilution 1:200 in antibody buffer). Several washing steps were followed by incubation with a cyanine 3-labeled secondary antibody (dilution 1:400 in antibody buffer; Jackson ImmunoResearch Labs, West Grove, PA). After extensive
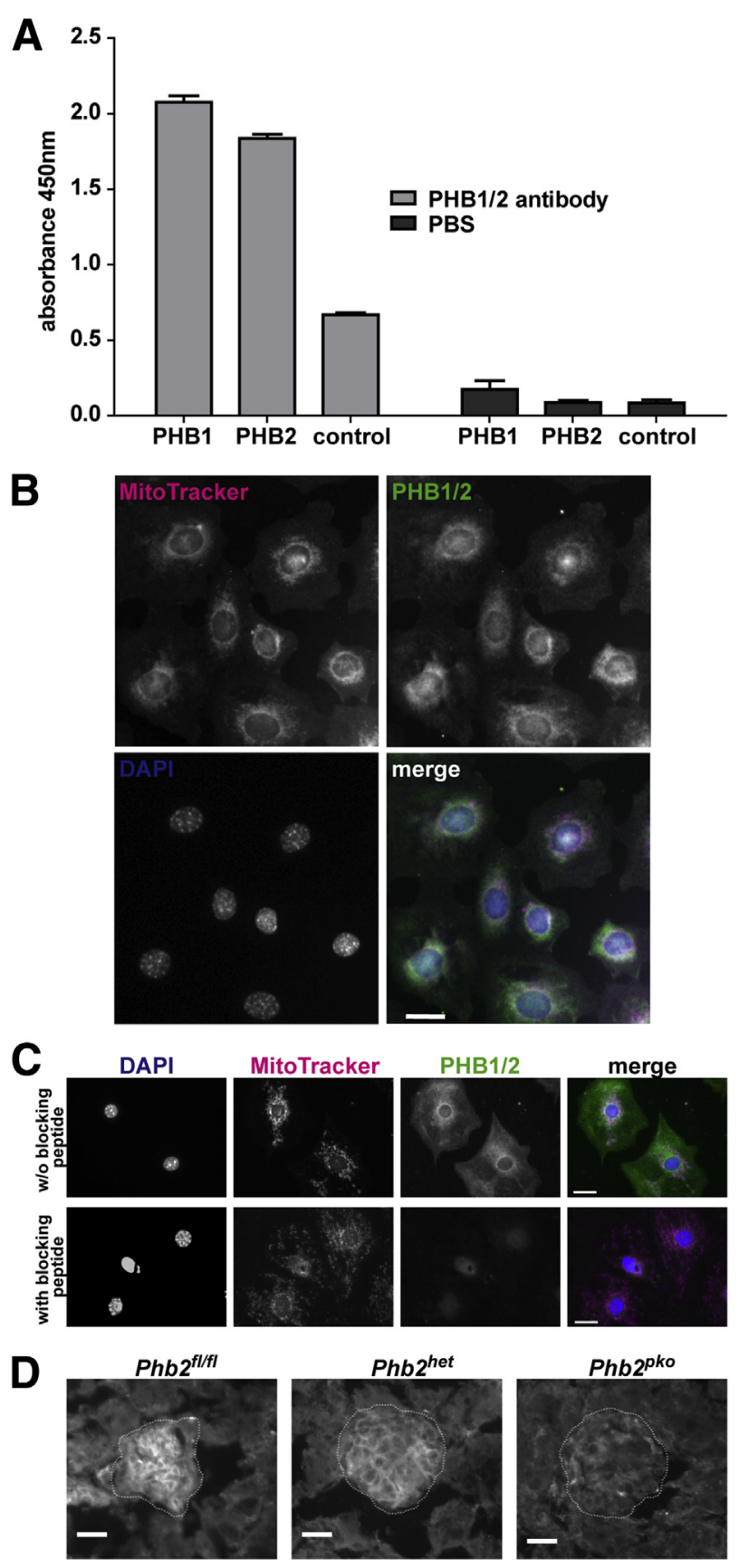

Figure 2 Validation of the PHB1/2 antibody. A: Enzyme-linked immunosorbent assay for PHB1, PHB2, and a control protein measured in triplicates. B: Costaining of MitoTracker and PHB1/2 on mouse podocytes. C: Costaining of MitoTracker and PHB1/2 on mouse podocytes with or without preincubation of PHB1/2 with recombinant PHB1 as blocking peptide. D: Staining of kidney sections of $P h b 2^{f l f l}, P h b 2^{h e t}$, and $P h b 2^{p k o}$ mice with PHB1/2 (dashed line circles surround a glomerulus). Data are expressed as means \pm SEM. Scale bar $=20 \mu \mathrm{m}$. PBS, phosphate-buffered saline; PHB, prohibitin; w/o, without.

washing the worms were subsequently counterstained with DAPI (Invitrogen) and mounted on slides with Prolong Gold Antifade Reagent (Invitrogen). Images were captured with a TCS SP8 X/PL Apo $100 \times / 1.4$ oil immersion objective operated by LAS X software (all from Leica Microsystems, Wetzlar, Germany). Images were 
further processed with ImageJ/Fiji software and Adobe Photoshop CS4.

\section{Statistical Analysis}

All results are expressed as means \pm SEM. Statistical significance was evaluated with GraphPad Prism version 4.00c for Macintosh (GraphPad Software, San Diego, CA). One-way analysis of variance with Tukey's posttest and two-way analysis of variance with Bonferroni's posttest were applied as indicated in the figure legends, and a $P<0.05$ was considered significant.

\section{Results}

PHB2 Is Required to Maintain Structural Integrity of Podocyte Foot Processes

Electron microscopy of 14-day-old podocyte-specific Phb2knockout $\left(P h b 2^{p k o}\right)$ mice revealed normal podocyte foot process organization and no overt changes in slit diaphragm appearance (Figure 1, A and B). During the third week of life $P h b 2^{p k o}$ mice developed foot process effacement and loss of slit diaphragm structures became evident, whereas foot processes of $P h b^{A / f t}$ and $P h b 2^{h e t}$ mice appeared normal (Figure 1, C and D).

As reported previously, ${ }^{17} \mathrm{Phb}^{\text {pko }}$ mice presented with disorganized mitochondrial cristae structures 3 weeks after birth (Figure 1, E and F). Interestingly, 21-day-old heterozygous littermates also showed altered mitochondrial ultrastructure, albeit to a lesser extent than $P h b 2^{p k o}$ mice and without any effect on animal survival or glomerular function (Figure 1, E and F).

Thus, although foot processes with proper slit diaphragm structures between neighboring podocytes were initially formed, $P h b 2^{p k o}$ mutants failed to maintain them over time, whereas $P h b 2^{\text {het }}$ mice showed normal slit diaphragm structures despite altered mitochondrial structure.

\section{PHB2 Localizes to the Slit Diaphragm}

The rapid loss of the slit diaphragm structure in $P h b 2^{p k o}$ mutants prompted us to investigate the subcellular localization of PHB2 in podocytes. To this end, we generated a novel monoclonal antibody that recognizes both PHB1 and PHB2 by immunofluorescence as shown by enzyme-linked immunosorbent assay (Figure 2A). We further tested our antibody in a costaining with MitoTracker on mouse podocytes in vitro (Figure 2B) and by preincubating the antibody with a blocking peptide (Figure 2C). Additional staining of mouse kidney sections $\left(P h b 2^{f l f t}, P h b 2^{h e t}\right.$, and $\left.P h b 2^{p k o}\right)$ further validated antibody specificity (Figure 2D). We also performed costaining of PHB1/2 and podocin on human kidney sections and observed not only the expected ubiquitous expression of PHB1/2 but also a sporadic overlap of PHB1/2 and podocin, indicating that at least one of the two PHB proteins potentially
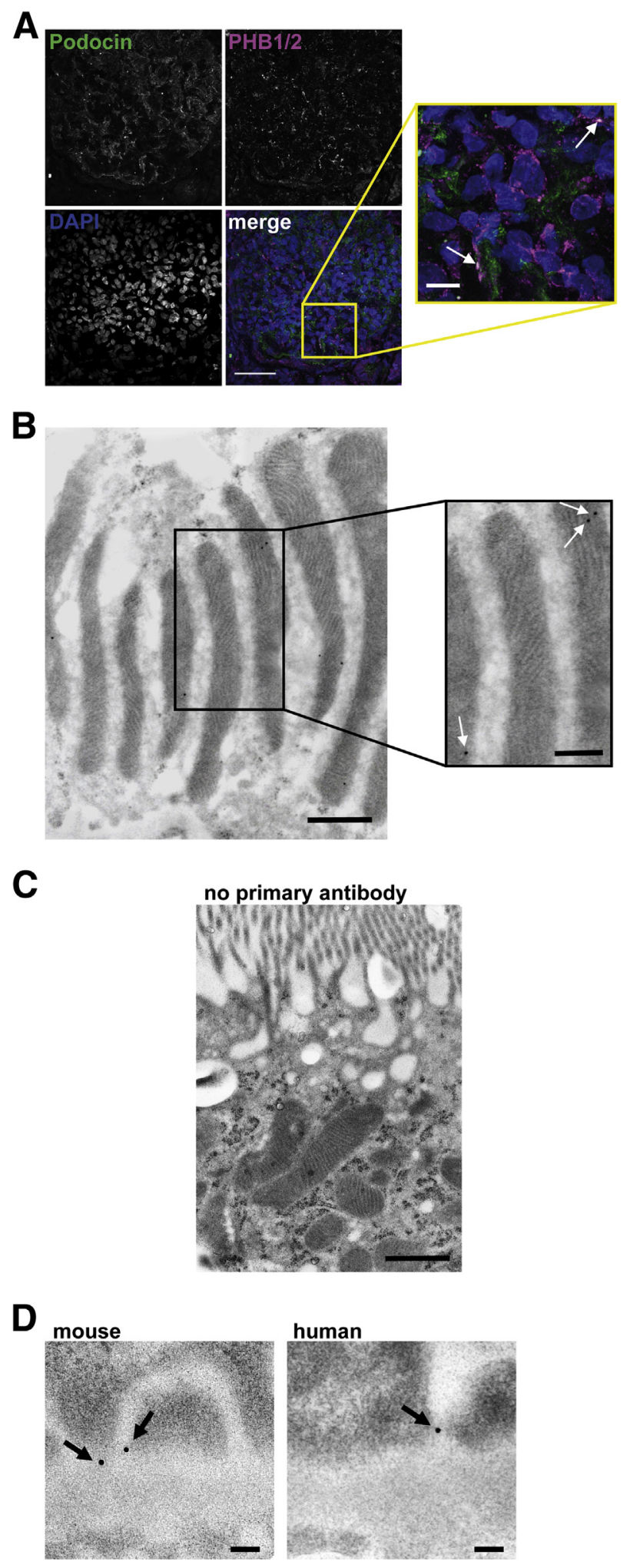

Figure 3 PHB2 localizes to mitochondria and partially to the slit diaphragm. A: Immunofluorescence staining of podocin and PHB1/2 on human kidney sections (arrows highlight the overlap). B: Immunogold labeling of PHB2 reveals signals in mitochondria on mouse kidney sections (arrows). C: Negative control of immunogold labeling (no primary antibody). D: Immunogold labeling of PHB2 at the slit diaphragm in mouse and human kidney sections (arrows). Scale bars: $50 \mu \mathrm{m}$ (A, main images), $10 \mu \mathrm{m}$ (A, magnification); 300nm (B and C); 150nm (B, magnification); 50nm (D). PHB, prohibitin. 

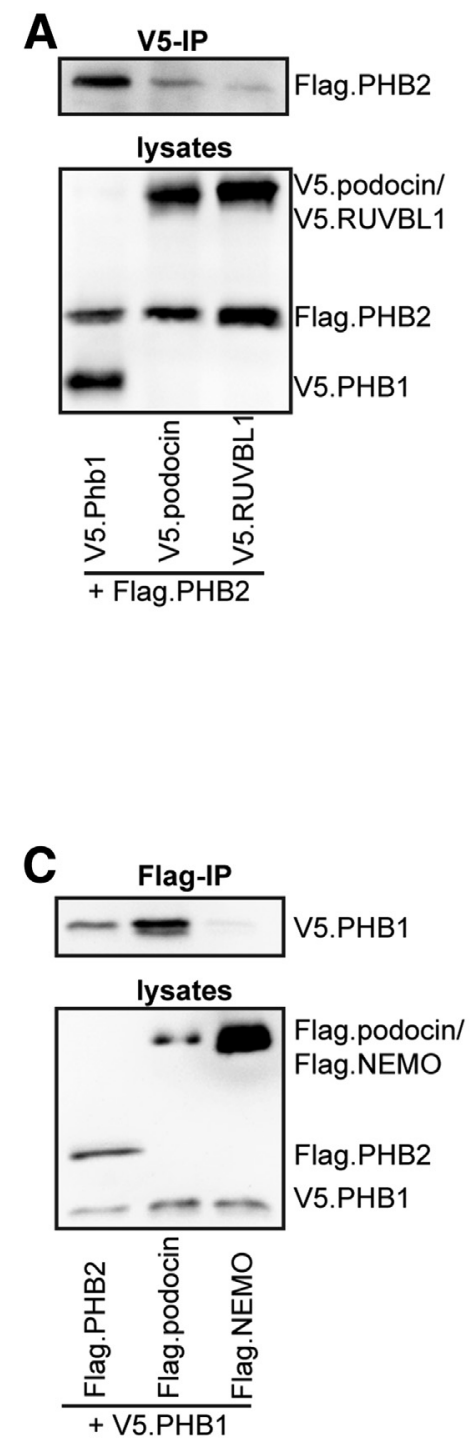
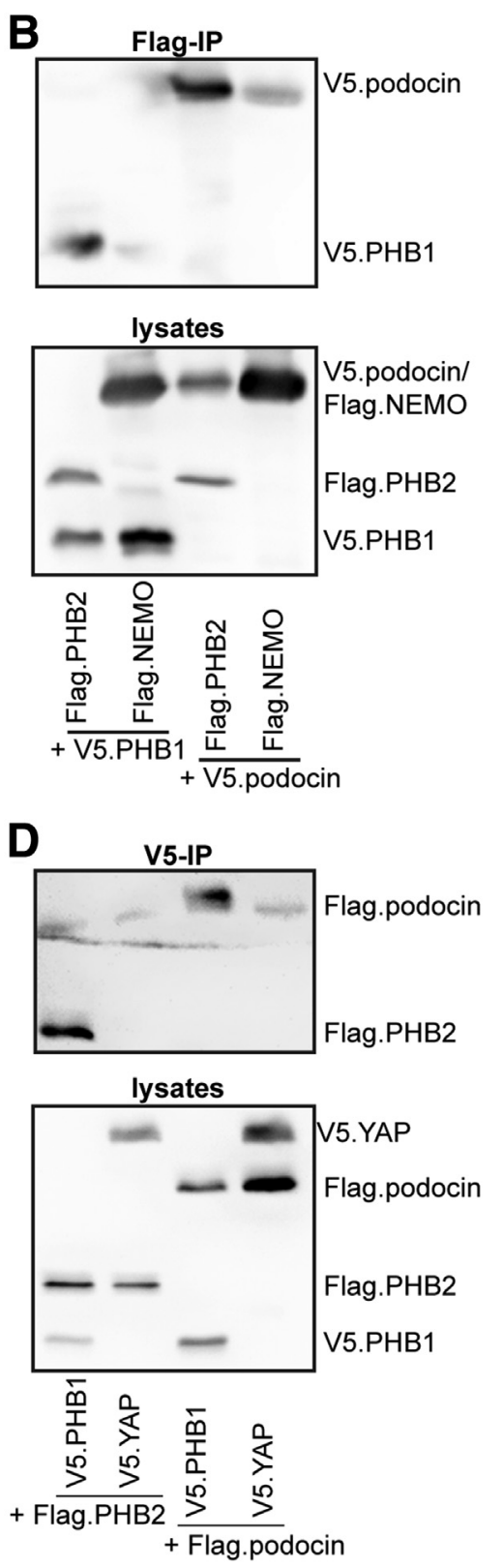

Figure 4 PHB1 and PHB2 coprecipitate with podocin. A-D: HEK293T cells were transiently transfected as indicated. IPs were performed with an anti-V5 antibody (A) or anti-Flag antibody (C). IPs were performed with an anti-Flag antibody (B) or anti-V5 antibody (D). E: Coimmunoprecipitation of podocin or a control antibody and PHB2 from isolated wild-type mouse glomeruli. $n=4$ for all experiments. IP, immunoprecipitation; PHB, prohibitin.
E
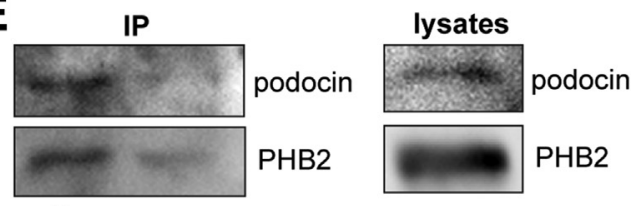

localizes to the slit diaphragm (Figure 3A). To further test this hypothesis we performed immunogold labeling on kidney sections with a commercially available PHB2-specific antibody. As expected, PHB2 was found to localize to mitochondria (Figure 3B); however, we did not see any signal in our negative control (Figure 3C). In addition, we also observed a specific staining at the slit diaphragm in normal kidney samples of mice and humans (Figure 3D).

Taken together, these results show that PHB2 localizes to the slit diaphragm in podocytes.

\section{PHB1 and PHB2 Coprecipitate with Podocin}

Co-immunoprecipitation experiments were performed to unravel a possible complex formation of PHB1/2 with the slit diaphragm protein podocin. First, we performed experiments in HEK293T cells that expressed epitopetagged PHB1 or PHB2 and podocin. The known interaction of PHB2 with PHB1 served as positive control, whereas the expression of a slit diaphragm-unrelated protein served as negative control. Here, we observed a specific co-immunoprecipitation of PHB1 and PHB2 with podocin but not with a control protein (Figure 4, A-D). In addition, immunoprecipitation of endogenously expressed podocin from isolated wild-type mouse glomeruli showed a specific coprecipitation of PHB2 with podocin (Figure 4E). These results suggest that PHB2 and possibly PHB1 as well do not only localize to the slit diaphragm but are also part of a protein complex with podocin.

Podocin was shown to form multimeric protein complexes at the plasma membrane and the multimerization requires the 

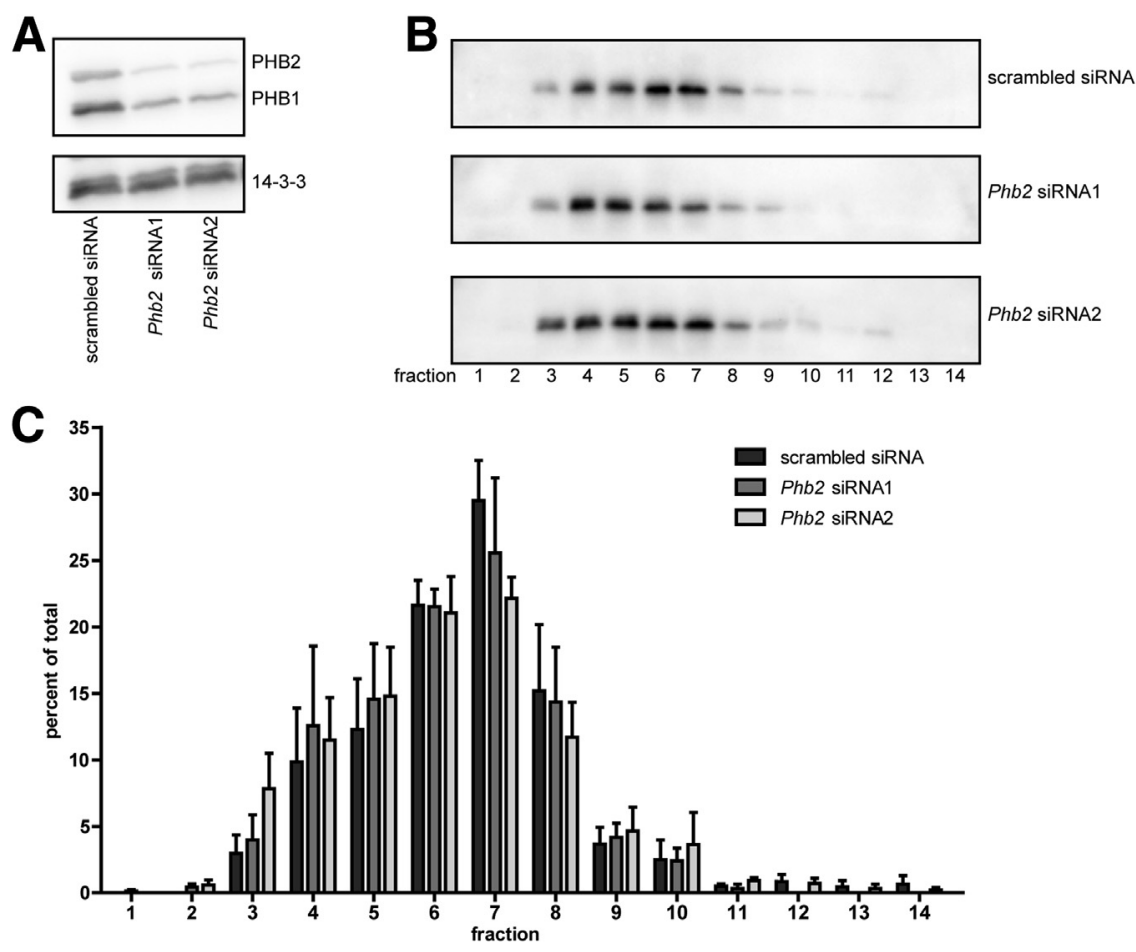

Figure 5 Podocin complexes show a trend to less multimeric complexes in Phb2-deficient cells. A: Western blot analysis of HEK293T cells 72 hours after transfection with Phb-2 siRNA1, Phb-2 siRNA2, or scrambled siRNA. B: Fraction 1 to 14 of velocity gradients stained for FLAG.podocin. C: Quantification of podocin levels in each fraction relative to total podocin amount. Data are expressed as means \pm SEM. $n=3 . P=1$ for treatment groups by two-way analysis of variance with Bonferroni's posttest. Phb, prohibitin. presence of the SPFH domain. ${ }^{34}$ The localization of PHB2 to the slit diaphragm and its co-immunoprecipitation with podocin suggest a role of the SPFH domain-containing protein PHB2 in the multimerization of podocin. To test this hypothesis we knocked down Phb2 in podocinoverexpressing HEK293T cells by using two different Phb2 siRNAs. Knockdown of Phb2 did not only lead to reduction of PHB2 protein levels but also of PHB1 protein levels (Figure 5A), as described previously. ${ }^{5}$ We subjected the cell lysates to velocity gradient centrifugations to assess podocin multimerization. Here, we did not observe any significant changes, albeit a slight trend to smaller podocin multimeric complexes in PHB2 knockdown cells (Figure 5, $\mathrm{B}$ and $\mathrm{C})$.

\section{PHB-2 Colocalizes with MEC-2 at Touch Receptor Punctae and Contributes to Touch Sensation in C. elegans}

As localization of PHB2 to the slit diaphragm and the interaction with podocin point to an additional, extra-mitochondrial role of PHB2 in podocytes, we tested the hypothesis that PHB2 affects the function of the slit diaphragm protein complex. Given the strong similarity of the mechanosensory machinery in $C$. elegans and the complex organization of the slit diaphragm in mammals, ${ }^{35,36}$ the mechanosensory complex in C. elegans is a well-established model to analyze the function of the slit diaphragm protein complex. ${ }^{35,37}$ The nematode ortholog of podocin, MEC-2, had been shown before to be an essential part of the nematode touch receptor complex and its physiologic function can easily be tested by gentle touch assays. ${ }^{38,39}$

Because a whole-body knockdown of phb-2 results in infertility and shortened life span, ${ }^{37}$ we used a cell-specific RNAi strain (sid-1(pk3321) him-5(e1490) V; lin-15B(n744) $X$; uIs71 [pCFJ90(myo-2p::mCherry) + mec-18p::sid-1]) in which the gene knockdown is restricted to the six touch receptor neurons without affecting the metabolism in any other cell. ${ }^{26}$ Knockdown of mec-2 led to impaired touch sensitivity as published previously ${ }^{31}$ and was included as control. Knockdown of either $p h b-1$ or $p h b-2$ resulted in a significant decrease in the touch responses (Figure 6A). This effect of PHB deficiency was not a consequence of a defective respiratory chain activity because we included $m e v-1$ RNAi as additional control. mev- 1 encodes for the succinate dehydrogenase cytochrome $b$; that is, the respiratory chain complex II and its knockdown causes disruption of mitochondrial respiration. ${ }^{40}$ Knockdown of mev-1 did not lead to a significant decrease in touch sensitivity compared with wild-type worms and could not recapitulate the effect of a $p h b-1$ or $p h b-2$ knockdown (Figure 6A).

The decreased touch sensitivity in $p h b-1-$ and $p h b-2-$ deficient worms prompted us to investigate whether PHB-2 might localize to touch punctae of mechanosensory neurons. Thus, we generated a transgenic strain that expressed PHB-2:GFP exclusively in the six touch receptor neurons. ${ }^{38}$ Immunofluorescence of the podocin homolog MEC-2 could confirm partial colocalization of PHB-2 and MEC-2 in the so-called touch punctae ${ }^{39}$ of these neurons (Figure 6B), which nicely complements our data from human and mouse kidneys. 
These results in C. elegans further confirmed our in vitro results and our in vivo results from the $P h b 2^{p k o}$ mouse model. Localization of PHB2 to the mechanosensoryprotein-super-complex appears to be evolutionarily

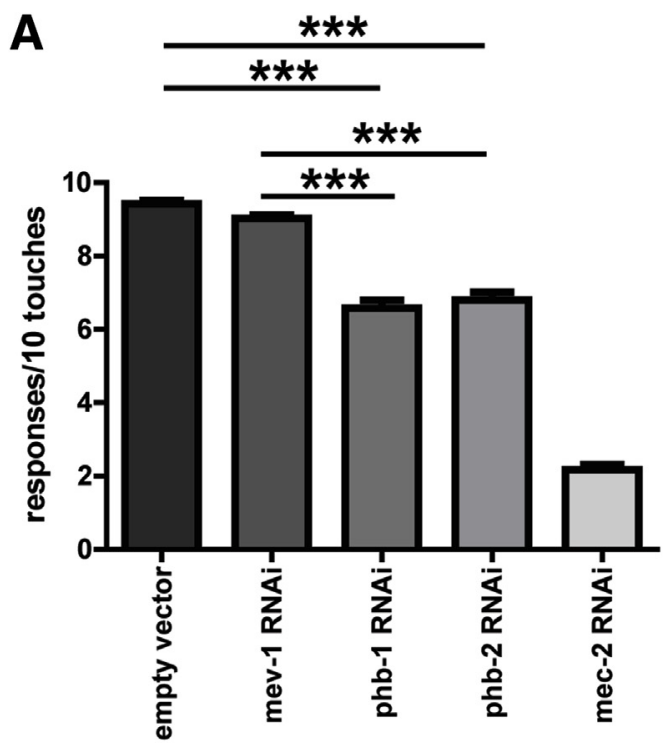

B worm1
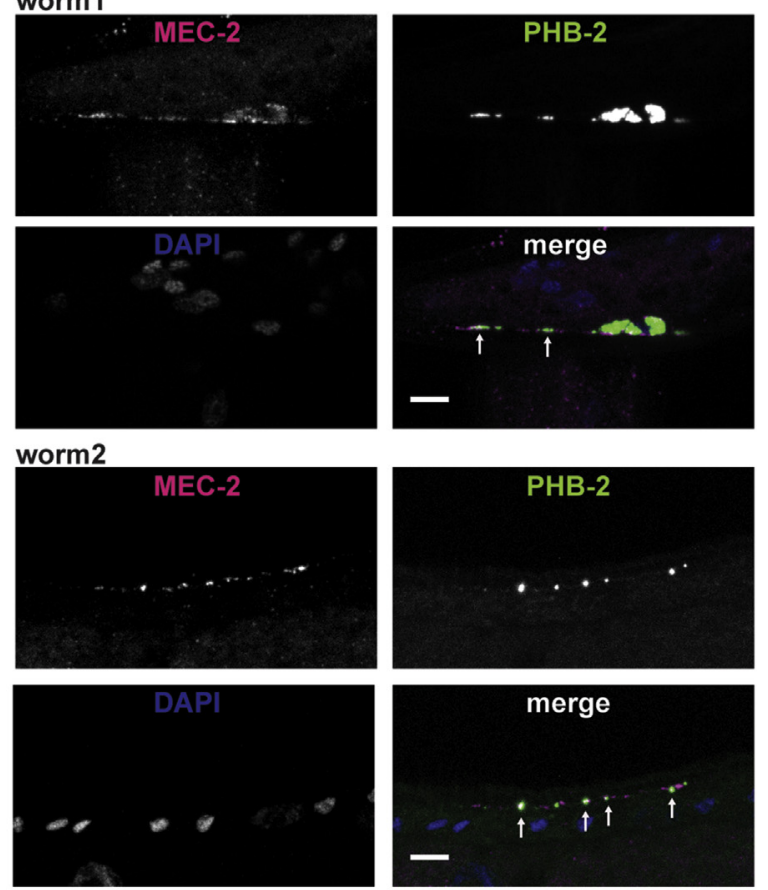

worm3
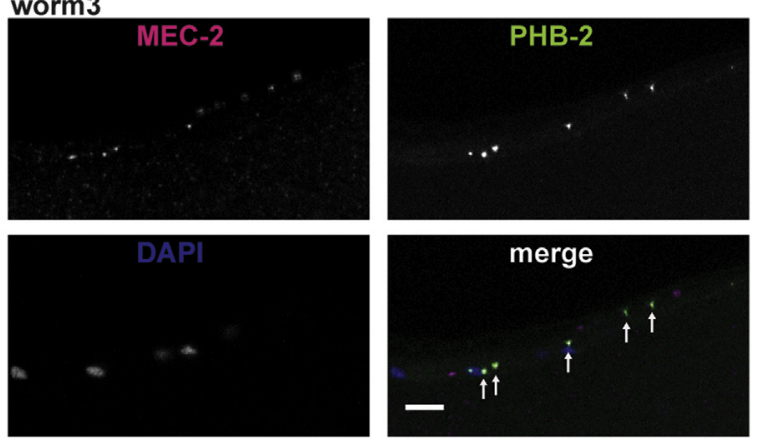

conserved, and its impact on mechanosensation is most likely independent of respiratory chain activity.

\section{Discussion}

Mitochondrial disorders remain enigmatic with only a poor correlation between gene defects and clinical symptoms. A number of mitochondriopathies present, among others, with renal symptoms, most often tubulopathies or podocyte dysfunction, leading to focal segmental glomerulosclerosis. $^{41}$ Lately, we studied the role of the structural mitochondrial protein PHB2 in podocytes. To this end, we generated a podocyte-specific $P h b 2$ knockout mouse strain $\left(P h b 2^{p k o}\right) .{ }^{17}$ At birth $P h b 2^{p k o}$ mutants appeared as healthy as wild-type or heterozygous controls. However, genetic deletion of PHB2 in podocytes led to the development of proteinuria, glomerulosclerosis, and premature death within 4 to 5 weeks. Although the inhibition of the insulin/insulinlike growth factor pathway alleviated renal disease and prolonged survival in $P h b 2$-knockout mice, the onset and levels of proteinuria inexplicably remained unaffected in these animals. ${ }^{17}$ Electron microscopic studies in $P h b 2^{\text {pko }}$ mice at 3 weeks of age revealed not only loss of mitochondrial structures but also a severe damage to the podocyte slit diaphragm as seen in patients with nephrotic syndromes.

Of note, the previously published phenotype of $P h b 2^{\text {pko }}$ mice $^{17}$ resembled at least partially the phenotype of some slit diaphragm protein knockout mice: CD2-associated protein-knockout mice developed proteinuria at day 14 , exhibited growth retardation starting 3 weeks after birth, and presented with glomerulosclerosis at about 4 weeks of age. $^{42,43}$ In contrast, nephrin- or podocin-knockout mice showed massive foot process effacement and rapidly developed proteinuria shortly after birth. Nephrinknockout mice died within 1 day after birth, and podocin-knockout animals died within the first 2 weeks. $^{44,45}$ To explain the differences in the onset of proteinuria and time of death it was speculated that other proteins might compensate for the function of CD2associated protein and podocin, leading to a slower time course of disease compared with nephrin-knockout mice. ${ }^{44}$

Figure 6 PHB-2 colocalizes with MEC-2 at touch receptor punctae and contributes to touch sensation in Caenorhabditis elegans. A: Touch sensitivity of $C$. elegans strain TU3568 fed with control RNAi, mev-1, phb-1, phb-2, or mec-2 RNAi (control RNAi $9.42 \pm 0.09$ responses/10 touches; mev-1 RNAi $9.02 \pm 0.10$ responses/10 touches; phb-1 RNAi $6.58 \pm 0.21$ responses/10 touches; phb-2 RNAi $6.80 \pm 0.21$ responses/10 touches; mec-2 RNAi $3.38 \pm 0.22$ responses/10 touches). B: Immunofluorescence staining of MEC-2 in PHB-2:GFP-expressing worms (arrows point at colocalization in touch receptor neurons; two independently generated PHB-2:GFP worm strains imaged). $n=50$ for all groups (A); $n>30$ (B). ${ }^{* * *} P<0.001$ for control RNAi versus $p h b-1$ RNAi, control RNAi versus phb-2 RNAi, mev-1 RNAi versus phb-1 RNAi, and mev-1 RNAi versus phb-2 RNAi by one-way analysis of variance with Tukey's posttest. Scale bar $=5 \mu \mathrm{m}$. PHB, prohibitin; RNAi, RNA interference. 


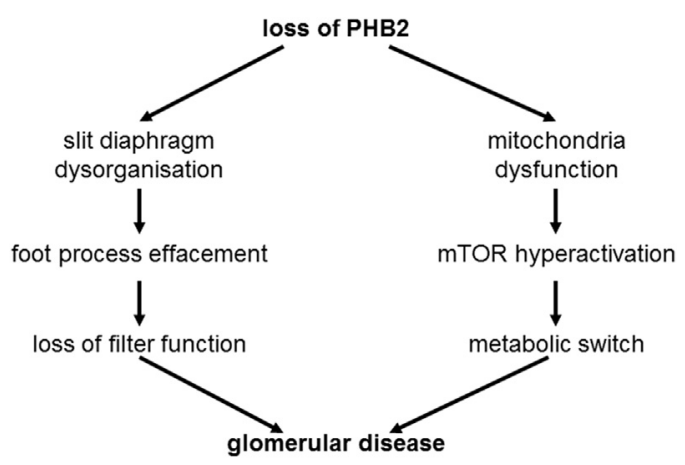

Figure 7 Suggested model for why loss of PHB2 leads to glomerular disease. Loss of PHB2 ultimately leads to glomerular disease potentially via two distinct pathways. On the one hand, PHB2 depletion results in foot process effacement because of its localization to the slit diaphragm, which results in loss of filter function (resulting in albuminuria). On the other hand, Phb2 deficiency leads to mitochondria dysfunction that enhances mTOR activation, resulting in a detrimental metabolic switch in podocytes (ultimately leading to podocyte loss). ${ }^{17}$ These two pathways together underlie the severe glomerular disease seen in $P h b 2^{p k o}$ mice. mTOR, mammalian target of rapamycin; PHB, prohibitin.

However, differences in genetic backgrounds could also account for these observations. ${ }^{45,46}$ Hence, we wondered how loss of the mitochondrial protein PHB2 could affect the filtering properties of podocytes to such a sustainable extent. In particular, as in heterozygous animals, in which we did not observe any overt phenotype, mitochondrial ultrastructure also appeared to be disturbed, albeit to a lesser extent than $P h b 2^{p k o}$ mice. Therefore, because PHB2 does not primarily affect electron transport chain maintenance and is not required for respiratory chain activity neither in mouse embryonic fibroblasts ${ }^{5}$ nor in isolated podocytes, ${ }^{17}$ we hypothesized that PHB2 confers additional, potentially extra-mitochondrial functions in podocytes. Immunofluorescence and immunogold labeling for PHB2 in glomeruli identified its subcellular distribution in podocytes. Here, we could observe a clear signal not only in mitochondria but also at the basis of the slit diaphragm, indicating that in addition to its localization to mitochondria PHB2 may also be part of the slit diaphragm complex.

Both podocin and PHBs belong to the SPFH domaincontaining protein family, and members of this family were shown to be able to build homoligomers and heteroligomers. ${ }^{2,47}$ Our findings of the localization of PHB2 to the slit diaphragm and its coprecipitation with podocin led to the hypothesis that PHBs could have a role in stabilizing the podocin-containing multimeric complexes at the slit diaphragm. However, we could not confirm this in our in vitro experiments with the use of velocity gradient centrifugation to isolate these multimerix complexes in the presence and absence of PHBs. These experiments are limited because we used Phb2-knockdown cells with residual expression of PHBs, which could still be sufficient to stabilize these complexes. Clearly, further work is necessary to shed light on the underlying mechanism.
Nevertheless, the observation of localization of PHB2 to the slit diaphragm raised the possibility that PHB2 may also affect the function of this specialized cell-cell contact. The mechanosensory machinery in the nematode $C$. elegans and the complex organization of the slit diaphragm in mammals are highly similar, with both podocin and its ortholog MEC-2 being essential for the formation of large proteincholesterol supercomplexes. ${ }^{35}$ Therefore, we used $C$. elegans to test for functionality of the multiprotein complex. We generated a transgenic strain in which expression of a PHB-2:GFP translational fusion was restricted to the six touch receptor neurons from late embryogenesis through adulthood. A similar overexpression approach was successfully established for PHB- $1{ }^{48}$ Staining of PHB-2:GFPoverexpressing worms for the podocin homolog MEC-2 revealed partial colocalization of both proteins in touch punctae. Furthermore, we performed gentle touch assays. Not only loss of mec-2 but also loss of both $p h b-1$ and $p h b-2$ led to a significant decrease in the touch responses, whereas knockdown of $m e v-1$, a protein of the respiratory chain, showed no significant effects on mechanosensation, further demonstrating that the observed effects are independent from the oxidative phosphorylation system as reported previously for podocytes. ${ }^{17}$

Clearly, further work is necessary to unravel the exact role of PHB2 at the slit diaphragm and to dissect it from its mitochondrial function in podocytes. There are certain limitations to our study. Studying SPFH proteins in general is technically demanding because these proteins easily undergo artificial protein-protein interactions in vitro and in particular when being tagged or studied in an overexpression system. Therefore, we took great care to include the appropriate controls and aimed at translating our findings to the in vivo situation by using the model organism C. elegans. However, we cannot exclude an epistatic interaction of $m e c-2$ and $p h b-2$ or additional effects of PHB2 on metabolic pathways, for example, lipid metabolism, which could influence the composition of the plasma membrane.

\section{Conclusions}

We provide several lines of evidence that suggest a mitochondria-independent function of PHB2 at the slit diaphragm. The identification of PHB2 as a second SPFH domain-containing protein at the slit diaphragm challenges our view of a podocin-multimeric-complex, which could also contain several other SPFH domain-containing proteins. A potential role of PHB2 at the slit diaphragm in mammals and at the mechanosensory complex in C. elegans appears to be evolutionarily conserved. Taking into account the results from this study and from our previously published study, ${ }^{17}$ we hypothesize a dual role for PHB2 in podocytes (Figure 7). On the one hand, loss of PHB2 from mitochondria accounts for the insulin/IGF-1-dependent 
premature death of $P h b 2^{p k o}$ mice as reported previously. ${ }^{17}$ On the other hand, loss of PHB2 at the slit diaphragm leads to the development of proteinuria in mice and perturbs mechanosensation in $C$. elegans, which is most likely independent of the function of PHB2 in mitochondria.

\section{Acknowledgments}

We thank Angelika Köser, Nadine Urban, Sonja Kunath, Katja Heckmann, Bettina Maar, Christian Hoffmann, Jessica Hausmann, Beatrix Martiny, and Brigitte Langer for excellent technical support; Martin Chalfie (Columbia University) for providing the neuronal-specific RNAi strain as well as the MEC-2 antibody; Stuart Shankland (University of Washington) for providing the immortalized mouse podocyte cell line; Rudolf Wiesner and Thomas Langer (University of Cologne) for helpful discussions; and the CECAD Imaging Facility for providing excellent microscopy infrastructure.

\section{References}

1. Back JW, Sanz MA, De Jong L, De Koning LJ, Nijtmans LG, De Koster CG, Grivell LA, Van Der Spek H, Muijsers AO: A structure for the yeast prohibitin complex: structure prediction and evidence from chemical crosslinking and mass spectrometry. Protein Sci 2002, 11: $2471-2478$

2. Tatsuta T, Model K, Langer T: Formation of membrane-bound ring complexes by prohibitins in mitochondria. Mol Biol Cell 2005, 16: 248-259

3. Merkwirth C, Langer T: Prohibitin function within mitochondria: essential roles for cell proliferation and cristae morphogenesis. Biochim Biophys Acta 2009, 1793:27-32

4. Kasashima K, Sumitani M, Satoh M, Endo H: Human prohibitin 1 maintains the organization and stability of the mitochondrial nucleoids. Exp Cell Res 2008, 314:988-996

5. Merkwirth C, Dargazanli S, Tatsuta T, Geimer S, Löwer B, Wunderlich FT, von Kleist-Retzow JC, Waisman A, Westermann B, Langer T: Prohibitins control cell proliferation and apoptosis by regulating OPA1-dependent cristae morphogenesis in mitochondria. Genes Dev 2008, 22:476-488

6. Osman C, Merkwirth C, Langer T: Prohibitins and the functional compartmentalization of mitochondrial membranes. J Cell Sci 2009, 122:3823-3830

7. Artal-Sanz M, Tavernarakis N: Prohibitin and mitochondrial biology. Trends Endocrinol Metab 2009, 20:394-401

8. Zhang L, Ji Q, Ni ZH, Sun J: Prohibitin induces apoptosis in BGC823 gastric cancer cells through the mitochondrial pathway. Asian Pac J Cancer Prev 2012, 13:3803-3807

9. Wu Q, Wu S: Lipid rafts association and anti-apoptotic function of prohibitin in ultraviolet B light-irradiated HaCaT keratinocytes. Exp Dermatol 2012, 21:640-642

10. Chowdhury I, Thompson WE, Welch C, Thomas K, Matthews R: Prohibitin (PHB) inhibits apoptosis in rat granulosa cells (GCs) through the extracellular signal-regulated kinase 1/2 (ERK1/2) and the Bcl family of proteins. Apoptosis 2013, 18:1513-1525

11. Chowdhury I, Thompson WE, Thomas K: Prohibitins role in cellular survival through Ras-Raf-MEK-ERK pathway. J Cell Physiol 2014, 229:998-1004

12. Wang S, Fusaro G, Padmanabhan J, Chellappan SP: Prohibitin colocalizes with $\mathrm{Rb}$ in the nucleus and recruits $\mathrm{N}-\mathrm{CoR}$ and HDAC1 for transcriptional repression. Oncogene 2002, 21:8388-8396
13. Fusaro G, Dasgupta P, Rastogi S, Joshi B, Chellappan S: Prohibitin induces the transcriptional activity of p53 and is exported from the nucleus upon apoptotic signaling. J Biol Chem 2003, 278:47853-47861

14. Kolonin MG, Saha PK, Chan L, Pasqualini R, Arap W: Reversal of obesity by targeted ablation of adipose tissue. Nat Med 2004, 10: $625-632$

15. Kurtev V, Margueron R, Kroboth K, Ogris E, Cavailles V, Seiser C: Transcriptional regulation by the repressor of estrogen receptor activity via recruitment of histone deacetylases. J Biol Chem 2004, 279: 24834-24843

16. Rajalingam K, Wunder C, Brinkmann V, Churin Y, Hekman M, Sievers C, Rapp UR, Rudel T: Prohibitin is required for Ras-induced Raf-MEK-ERK activation and epithelial cell migration. Nat Cell Biol 2005, 7:837-843

17. Ising C, Koehler S, Brähler S, Merkwirth C, Höhne M, Baris OR, Hagmann H, Kann M, Fabretti F, Dafinger C, Bloch W, Schermer B, Linkermann A, Brüning JC, Kurschat CE, Müller RU, Wiesner RJ, Langer T, Benzing T, Brinkkoetter PT: Inhibition of insulin/IGF-1 receptor signaling protects from mitochondria-mediated kidney failure. EMBO Mol Med 2015, 7:275-287

18. Boute N, Gribouval O, Roselli S, Benessy F, Lee H, Fuchshuber A, Dahan K, Gubler MC, Niaudet P, Antignac C: NPHS2, encoding the glomerular protein podocin, is mutated in autosomal recessive steroid-resistant nephrotic syndrome. Nat Genet 2000, 24:349-354

19. Moeller MJ, Sanden SK, Soofi A, Wiggins RC, Holzman LB: Podocyte-specific expression of cre recombinase in transgenic mice. Genesis 2003, 35:39-42

20. Horvat R, Hovorka A, Dekan G, Poczewski H, Kerjaschki D: Endothelial cell membranes contain podocalyxin-the major sialoprotein of visceral glomerular epithelial cells. J Cell Biol 1986, 102:484-491

21. Griffin SV, Olivier JP, Pippin JW, Roberts JM, Shankland SJ: Cyclin I protects podocytes from apoptosis. J Biol Chem 2006, 281: 28048-28057

22. Shankland SJ, Pippin JW, Reiser J, Mundel P: Podocytes in culture: past, present, and future. Kidney Int 2007, 72:26-36

23. Köhler G, Milstein C: Continuous cultures of fused cells secreting antibody of predefined specificity. Nature 1975, 256:495-497

24. Benzing T, Gerke P, Höpker K, Hildebrandt F, Kim E, Walz G: Nephrocystin interacts with Pyk2, p130(Cas), and tensin and triggers phosphorylation of Pyk2. Proc Natl Acad Sci U S A 2001, 98 : 9784-9789

25. Brähler S, Ising C, Hagmann H, Rasmus M, Hoehne M, Kurschat C, Kisner $\mathrm{T}$, Goebel $\mathrm{H}$, Shankland $\mathrm{S}$, Addicks $\mathrm{K}$, Thaiss $\mathrm{F}$, Schermer B, Pasparakis M, Benzing T, Brinkkoetter PT: Intrinsic proinflammatory signaling in podocytes contributes to podocyte damage and prolonged proteinuria. Am J Physiol Renal Physiol 2012, 303:F1473-F1485

26. Calixto A, Chelur D, Topalidou I, Chen X, Chalfie M: Enhanced neuronal RNAi in C. elegans using SID-1. Nat Methods 2010, 7: $554-559$

27. Brenner S: The genetics of Caenorhabditis elegans. Genetics 1974, 77: $71-94$

28. Fire A, Xu S, Montgomery MK, Kostas SA, Driver SE, Mello CC: Potent and specific genetic interference by double-stranded RNA in Caenorhabditis elegans. Nature 1998, 391:806-811

29. Kamath RS, Ahringer J: Genome-wide RNAi screening in Caenorhabditis elegans. Methods 2003, 30:313-321

30. Artal-Sanz M, Tsang WY, Willems EM, Grivell LA, Lemire BD, van der Spek H, Nijtmans LG: The mitochondrial prohibitin complex is essential for embryonic viability and germline function in Caenorhabditis elegans. J Biol Chem 2003, 278:32091-32099

31. Chalfie M, Sulston J: Developmental genetics of the mechanosensory neurons of Caenorhabditis elegans. Dev Biol 1981, 82:358-370

32. Finney M, Ruvkun G: The unc-86 gene product couples cell lineage and cell identity in C. elegans. Cell 1990, 63:895-905 
33. Bharill P, Ayyadevara S, Alla R, Shmookler Reis RJ: Extreme depletion of PIP3 accompanies the increased life span and stress tolerance of PI3K-null C. elegans mutants. Front Genet 2013, 4:34

34. Huber TB, Simons M, Hartleben B, Sernetz L, Schmidts M, Gundlach E, Saleem MA, Walz G, Benzing T: Molecular basis of the functional podocin-nephrin complex: mutations in the NPHS2 gene disrupt nephrin targeting to lipid raft microdomains. Hum Mol Genet 2003, 12:3397-3405

35. Huber TB, Schermer B, Müller RU, Höhne M, Bartram M, Calixto A, Hagmann H, Reinhardt C, Koos F, Kunzelmann K, Shirokova E, Krautwurst D, Harteneck C, Simons M, Pavenstädt H, Kerjaschki D, Thiele C, Walz G, Chalfie M, Benzing T: Podocin and MEC-2 bind cholesterol to regulate the activity of associated ion channels. Proc Natl Acad Sci U S A 2006, 103:17079-17086

36. Schermer B, Benzing T: Lipid-protein interactions along the slit diaphragm of podocytes. J Am Soc Nephrol 2009, 20:473-478

37. Müller RU, Zank S, Fabretti F, Benzing T: Caenorhabditis elegans, a model organism for kidney research: from cilia to mechanosensation and longevity. Curr Opin Nephrol Hypertens 2011, 20:400-408

38. Zhang Y, Ma C, Delohery T, Nasipak B, Foat BC, Bounoutas A, Bussemaker HJ, Kim SK, Chalfie M: Identification of genes expressed in C. elegans touch receptor neurons. Nature 2002, 418:331-335

39. Zhang S, Arnadottir J, Keller C, Caldwell GA, Yao CA, Chalfie M: MEC-2 is recruited to the putative mechanosensory complex in C. elegans touch receptor neurons through its stomatin-like domain. Curr Biol 2004, 14:1888-1896

40. Ishii N, Fujii M, Hartman PS, Tsuda M, Yasuda K, Senoo-Matsuda N, Yanase S, Ayusawa D, Suzuki K: A mutation in succinate dehydrogenase cytochrome b causes oxidative stress and ageing in nematodes. Nature 1998, 394:694-697

41. Che R, Yuan Y, Huang S, Zhang A: Mitochondrial dysfunction in the pathophysiology of renal diseases. Am J Physiol Renal Physiol 2014, 306:F367-F378

42. Shih NY, Li J, Karpitskii V, Nguyen A, Dustin ML, Kanagawa O, Miner JH, Shaw AS: Congenital nephrotic syndrome in mice lacking CD2-associated protein. Science 1999, 286:312-315

43. Shih NY, Li J, Cotran R, Mundel P, Miner JH, Shaw AS: CD2AP localizes to the slit diaphragm and binds to nephrin via a novel Cterminal domain. Am J Pathol 2001, 159:2303-2308

44. Putaala H, Soininen R, Kilpeläinen P, Wartiovaara J, Tryggvason K: The murine nephrin gene is specifically expressed in kidney, brain and pancreas: inactivation of the gene leads to massive proteinuria and neonatal death. Hum Mol Genet 2001, 10:1-8

45. Roselli S, Heidet L, Sich M, Henger A, Kretzler M, Gubler MC, Antignac C: Early glomerular filtration defect and severe renal disease in podocin-deficient mice. Mol Cell Biol 2004, 24:550-560

46. Ma LJ, Fogo AB: Model of robust induction of glomerulosclerosis in mice: importance of genetic background. Kidney Int 2003, 64: 350-355

47. Snyers L, Umlauf E, Prohaska R: Oligomeric nature of the integral membrane protein stomatin. J Biol Chem 1998, 273: $17221-17226$

48. Artal-Sanz M, Tavernarakis N: Prohibitin couples diapause signalling to mitochondrial metabolism during ageing in C. elegans. Nature 2009, 461:793-797 